\title{
TWO MASSIVE STAR-FORMING REGIONS AT EARLY EVOLUTIONARY STAGES
}

\author{
Guido Garay \\ Departamento de Astronomía, Universidad de Chile, Casilla 36-D, Santiago, Chile \\ KATE J. BROOKS \\ European Southern Observatory, Casilla 19001, Santiago 19, Chile \\ Diego Mardones \\ Departamento de Astronomía, Universidad de Chile, Casilla 36-D, Santiago, Chile \\ RAY P. NORRIS \\ Australia Telescope National Facility, P.O. Box 76, Epping, NSW 1710, Australia \\ AND \\ Michael G. Burton \\ Department of Astrophysics and Optics, School of Physics, University of New South Wales, Sydney, NSW 2052, Australia \\ Received 2002 May24; accepted 2002 July 18
}

\begin{abstract}
We report sensitive Australia Telescope Compact Array radio continuum observations toward IRAS 15596-5301 and 16272-4837, two luminous objects $\left(L>2 \times 10^{4} L_{\odot}\right)$ thought to represent massive starforming regions in early stages of evolution (due to previously undetected radio emission at the $1 \sigma$ level of $2 \mathrm{mJy}$ beam $^{-1}$ ). Also reported are $1.2 \mathrm{~mm}$ continuum and a series of molecular line observations made with the Swedish ESO Submillimeter Telescope. The radio continuum observations toward IRAS 15596-5301 reveal the presence of three distinct compact sources, with angular sizes of 2!"7-8".8 (FWHM), all located within a region of $30^{\prime \prime}$ in diameter. Assuming that these are regions of ionized gas, we find that they have diameters of $0.06-0.2 \mathrm{pc}$ and electron densities of $8 \times 10^{2}-2 \times 10^{3} \mathrm{~cm}^{-3}$ and that they are excited by early B-type stars. The $1.2 \mathrm{~mm}$ observations show that the dust emission arises from a region of $42^{\prime \prime} \times 25^{\prime \prime}$ (FWHM) with a total flux of $5.8 \mathrm{Jy}$, implying a mass of $1.4 \times 10^{3} M_{\odot}$. The line observations indicate that IRAS 15596-5301 is associated with a molecular cloud with a FWHM angular size of $37^{\prime \prime}(\sim 0.4 \mathrm{pc}$ radius at the distance of $4.6 \mathrm{kpc}$ ), a molecular hydrogen density of $\sim 4 \times 10^{5} \mathrm{~cm}^{-3}$, and a rotational temperature of $\sim 27 \mathrm{~K}$. We suggest that the massive dense core associated with IRAS 15596-5301 contains a cluster of $\mathrm{B}$ stars that are exciting compact $\mathrm{H}$ II regions that are in pressure equilibrium with the dense molecular surroundings. No radio continuum emission was detected from IRAS $16272-4837$ up to a $3 \sigma$ limit of $0.2 \mathrm{mJy}$. However, the $1.2 \mathrm{~mm}$ observations show strong dust emission arising from a region of $41^{\prime \prime} \times 25^{\prime \prime}(\mathrm{FWHM})$ with a total flux of $13.8 \mathrm{Jy}$, implying a mass of $2.0 \times 10^{3} M_{\odot}$. The line observations indicate the presence of an elongated molecular cloud with FWHM major and minor axes of $61^{\prime \prime}$ and $42^{\prime \prime}(0.50 \times 0.35 \mathrm{pc}$ in radius at the distance of $3.4 \mathrm{kpc}$ ), a molecular hydrogen density of $\sim 2 \times 10^{5} \mathrm{~cm}^{-3}$, and a rotational temperature of $\sim 27 \mathrm{~K}$. The high luminosity $\left(2.4 \times 10^{4} L_{\odot}\right)$ and lack of radio emission from this massive core suggest that it hosts an embedded young massive protostar that is still undergoing an intense accretion phase. This scenario is supported by the observed characteristics of the line profiles and the presence of a bipolar outflow detected from observations of the $\mathrm{SiO}$ emission. We suggest that IRAS 16272-4837 is a bona fide massive starforming region in a very early evolutionary stage, being the precursor of an ultracompact $\mathrm{H}$ in region.
\end{abstract}

Subject headings: H II regions — ISM: individual (IRAS 15596-5301, IRAS 16272-4837) — stars: early-type — stars: formation

\section{INTRODUCTION}

The earliest phase of high-mass star formation is possibly one of the least understood stages of evolution of massive stars. Massive stars $\left(M>8 M_{\odot}\right)$ are known to be formed in dense molecular cores; however, the sequence of processes leading to their formation is not yet well established. In particular, the roles of coalescence (Stahler, Palla, \& Ho 2000) and accretion (Osorio, Lizano, \& D'Alessio 1999) processes in the assembling of a massive star are still under debate. The determination of the physical conditions of the gas during the formation and early evolution of massive stars is difficult because of their scarcity and rapid evolution. In addition, massive stars are usually born in clusters or groups; hence, their individual studies are usually afflicted with confusion, particularly because they are found located, on the average, at larger distances from the Sun than sites of low-mass star formation. Massive objects in early evolutionary stages, namely, in the process of assembling the bulk of their final mass, should be identified by having high bolometric luminosities $\left(>10^{4} L_{\odot}\right)$, strong dust emission, and very weak or no detectable free-free emission at centimeter radio wavelengths. The bolometric luminosity has contributions from the accretion of infalling material and nuclear burning. Up to the present, only a few systematic searches for high-mass protostellar objects have been carried out (Molinari et al. 1996, 1998, 2000; Sridharan et al. 2002).

We have recently started a multiwavelength study of a sample of 18 luminous IRAS sources in the southern hemisphere thought to be representative of young mas- 
sive star-forming regions (D. Mardones, G. Garay, \& L. Bronfman 2002, in preparation). The goal is to understand the physical and chemical differences between different stages of early evolution. The objects were taken from the Galaxy-wide survey of $\mathrm{CS}(2 \rightarrow 1)$ emission toward IRAS sources with IR colors typical of compact H II regions (Bronfman, Nyman, \& May 1996). We selected sources based primarily on the observed $\mathrm{CS}(2 \rightarrow 1)$ line profiles, looking for self-absorbed lines consistent with inward or outward motions (e.g., Mardones 1998) and/or with extended line wings, possibly indicating the presence of bipolar outflows. In addition, the sources were required to have IRAS $100 \mu$ m fluxes greater than $10^{3} \mathrm{Jy}$ and to be in the southern hemisphere $\left(\delta<-20^{\circ}\right)$. The luminosity of the IRAS sources, computed using the IRAS energy distribution and the distances derived by L. Bronfman (2002, private communication), are in the range $2 \times 10^{4}-4 \times 10^{5} L_{\odot}$, implying that they contain at least one embedded massive star.

Most of the selected objects are expected to be associated with ultracompact (UC) $\mathrm{H}$ II regions, which are thought to be manifestations of newly formed massive stars that are still embedded in their natal molecular cloud. This expectation is confirmed by the radio continuum observations of Walsh et al. (1998), which show that nine of the 12 sources in both samples have detectable radio continuum emission (above a $3 \sigma$ limit of $6 \mathrm{mJy}$ beam $^{-1}$ at $8.64 \mathrm{GHz}$ with an angular resolution of $\sim 1^{\prime \prime}$ ). The objects that were not detected at radio wavelengths are suitable candidates for massive stars in very early stages of evolution in which dense material is still falling toward a massive OB-type protostar. In this accretion phase, the high mass accretion rate of the infalling material quenches the development of a UC H II region (Yorke 1984; Walmsley 1995), and the free-free emission from the ionized material is undetectable at centimeter wavelengths. The mass accretion rate might also be large enough that the ram pressure of the infalling gas could provide the force to prevent the expansion of an $\mathrm{H}$ II region. We note, however, that due to the limited sensitivity of the Walsh et al. (1998) survey, low-density H II regions with emission measures smaller than $4 \times 10^{5} \mathrm{pc}$ $\mathrm{cm}^{-6}$ were not detectable. Hence, the lack of detection at the above limit does not rule out the presence of an optically thin compact $\mathrm{H}$ II region.

In this paper we report sensitive Australia Telescope Compact Array (ATCA) radio continuum observations toward two sources in our sample, IRAS 15596-5301 and 16272-4837, without previously detected radio continuum emission to place stringent limits on their radio flux density. The $1 \sigma$ sensitivity level of $70 \mu \mathrm{Jy}$ at $4.8 \mathrm{GHz}$ achieved in our observations is 30 times smaller than in previous studies and is sufficient to detect the emission measure corresponding to any ionizing OB star within the Galaxy. The main goal is to establish whether or not these objects correspond to very young massive objects: that is, massive protostars that have not yet ionized significant amounts of the surrounding gas. Also reported in this paper are millimeter continuum and molecular line observations of IRAS 15596-5301 and 16272-4837 made with the Swedish ESO Submillimeter Telescope (SEST). The latter observations are part of a molecular line survey toward several high-mass star-forming regions made in order to determine their physical characteristics and investigate possible chemical differences.

\section{OBSERVATIONS}

The observations were made using ATCA $^{1}$ in Australia and the $15 \mathrm{~m}$ SEST located in La Silla, Chile.

\subsection{ATCA}

The ATCA radio continuum observations were made in 2000 May 23, using the 6D configuration, which utilizes all six antennas and covers east-west baselines from $77 \mathrm{~m}$ to 5.9 $\mathrm{km}$. Observations were made simultaneously at the frequencies of 4.800 and $4.928 \mathrm{GHz}$, each spanning a bandwidth of $128 \mathrm{MHz}$. At these frequencies, the FWHM primary beam of ATCA is $10^{\prime}$. The total integration time for each source was 270 minutes, obtained from 15 minute scans taken over a wide range of hour angles to provide good $(u, v)$-plane coverage. The calibrator PKS 1600-48 was observed before and after every on-source scan in order to correct the amplitude and phase of the interferometer data for atmospheric and instrumental effects. The flux density was calibrated by observing PKS 1934-638 (3C84) for which values of 5.83 Jy at $4.8 \mathrm{GHz}$ and $5.65 \mathrm{Jy}$ at $4.9 \mathrm{GHz}$ were adopted. Standard calibration and data reduction were performed using MIRIAD (Sault, Teuben, \& Wright 1995). Maps were made by Fourier transformation of the uniformly weighted interferometer data using the AIPS task MX. The noise level in each image is $70 \mu \mathrm{Jy}$, close to the expected theoretical limit of $50 \mu \mathrm{Jy}$. The synthesized (FWHM) beams were 2 ". $30 \times 1$ "'.68 for IRAS $15596-5301$ and 2 ". $46 \times 1$ ". 64 for IRAS $16272-4837$.

\subsection{SEST \\ 2.2.1. Millimeter Continuum}

The $1.2 \mathrm{~mm}$ continuum observations were made in 2001 September using the 37 channel SEST Imaging Bolometer Array (SIMBA). The half-power beamwidth of a single element is $24^{\prime \prime}$, and the separation between elements on the sky is $44^{\prime \prime}$. We made the observations in the fast-mapping mode, using a scan speed of $80^{\prime \prime} \mathrm{s}^{-1}$. Our observing blocks consisted of 50 scan lines in azimuth of length $800^{\prime \prime}$ and separated in elevation by $8^{\prime \prime}$, giving a map size in elevation of $400^{\prime \prime}$. This block required $\sim 15$ minutes of observing time. Two blocks were observed for each source. The data were reduced in a standard fashion, applying sky-opacity correction, calibration using a counts-to-flux conversion factor derived from maps of bright planets, baseline subtraction, and rejection of correlated sky noise using the software package MOPSI. The rms noise level in the final maps is 0.1 Jy, with a pixel binning of $8^{\prime \prime}$. Uncertainties in the pointing accuracy are estimated at $1 \sigma=5^{\prime \prime}$.

\subsubsection{Molecular Lines}

The molecular line observations were carried out in two epochs. During 1999 June, we used as a back end the high-resolution acousto-optical spectrometers, which provided a channel separation of $43 \mathrm{kHz}$ and a total bandwidth of $43 \mathrm{MHz}$. We observed, at the position of the IRAS sources, molecular line emission in 10 transitions: $\mathrm{CS}(2 \rightarrow 1), \quad \mathrm{CS}(5 \rightarrow 4), \quad \mathrm{CS}(7 \rightarrow 6), \quad \mathrm{C}^{34} \mathrm{~S}(2 \rightarrow 1)$, $\mathrm{SiO}(2 \rightarrow 1), \mathrm{SO}\left(6_{5} \rightarrow 5_{4}\right), \mathrm{HCO}^{+}(1 \rightarrow 0), \mathrm{HCO}^{+}(3 \rightarrow 2)$, $\mathrm{H}^{13} \mathrm{CO}^{+}(1 \rightarrow 0)$, and $\mathrm{C}^{18} \mathrm{O}(2 \rightarrow 1)$, with integration times

\footnotetext{
${ }^{1}$ The Australia Telescope Compact Array is funded by the Commonwealth of Australia for operation as a national facility managed by CSIRO.
} 
on source ranging from 3 to 6 minutes. System temperatures were typically $\sim 220 \mathrm{~K}$ at $3 \mathrm{~mm}, \sim 350 \mathrm{~K}$ at $1 \mathrm{~mm}$, and $\sim 700$ $\mathrm{K}$ at $0.8 \mathrm{~mm}$. In addition, we mapped, with angular spacings of $30^{\prime \prime}$, the $\operatorname{CS}(2 \rightarrow 1)$ and $\operatorname{CS}(5 \rightarrow 4)$ emission within regions of 2.5 and the $\mathrm{SiO}(2 \rightarrow 1)$ emission within regions of $2.5 \times 1.5$, centered on the IRAS positions. These observations were made in order to investigate the kinematics of the molecular gas.

During 2000 March, we made a survey of molecular line emission using as a back end the low-resolution acoustooptical spectrometers providing a channel separation of 700 $\mathrm{kHz}$ and a total bandwidth of $1.0 \mathrm{GHz}$. The $100 / 150 \mathrm{GHz}$ and $115 / 230 \mathrm{GHz}$ pairs of solid-state imaging spectrometer receivers were used to simultaneously observe lines in, respectively, the $3 / 2 \mathrm{~mm}$ and $3 / 1 \mathrm{~mm}$ wavelength ranges. We observed 39 spectral windows, $1.0 \mathrm{GHz}$ wide each, within the $85-250 \mathrm{GHz}$ frequency range. System temperatures were typically $\sim 200 \mathrm{~K}$ at 2 and $3 \mathrm{~mm}$, and $\sim 400 \mathrm{~K}$ at 1 $\mathrm{mm}$. The integration times on source were 5 minutes, resulting in antenna temperature rms noises of typically $20 \mathrm{mK}$. A detailed description of these observations is given in G. Garay, J. Pineda, \& D. Mardones (2002, in preparation). All the observations were performed in the dual beamswitching mode, with a beam separation of $11^{\prime} 47^{\prime \prime}$ in azimuth.

\section{RESULTS}

\subsection{Radio Continuum Emission}

Figure 1 shows a map of the $6 \mathrm{~cm}$ radio continuum emission from IRAS 15596-5301. Three compact radio sources (labeled A, B, and C) were detected within a region of $30^{\prime \prime}$ in diameter. Their positions, flux densities, and sizes are given in Table 1. Their peak flux densities are in the range 1.1-2.8 $\mathrm{mJy}$ beam $^{-1}$, close to the sensitivity limit in the survey of

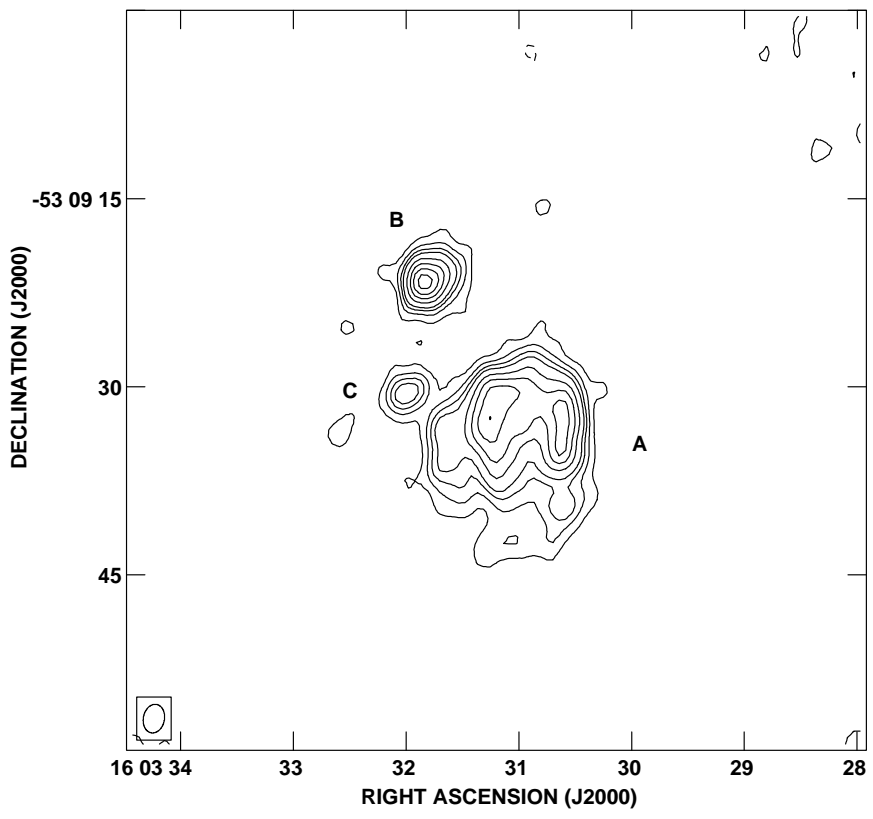

FIG. 1.-ATCA map of the $6 \mathrm{~cm}$ radio continuum emission from IRAS 15596-5301. The angular resolution is 2 ". $30 \times 1$ ".68 (FWHM beam is shown in the bottom left corner). Contour levels are $-3,3,6,9,12,18,24$, 30 , and 36 times $70 \mu \mathrm{Jy}$ beam $^{-1}$ ( $=1 \sigma$ noise level).
TABLE 1

Observed Parameters of Radio Continuum Emission

\begin{tabular}{|c|c|c|c|c|}
\hline \multirow[b]{2}{*}{ H II ReGion } & \multicolumn{2}{|c|}{ Position } & \multirow{2}{*}{$\begin{array}{c}\text { FLUX } \\
\text { DENSITY } \\
(\mathrm{mJy})\end{array}$} & \multirow{2}{*}{$\begin{array}{c}\text { ANGULAR } \\
\text { SIZE } \\
(\operatorname{arcsec})\end{array}$} \\
\hline & $\alpha(\mathrm{J} 2000.0)$ & $\delta(\mathbf{J} 2000.0)$ & & \\
\hline А & 160331.01 & -530932.9 & 43.4 & $9.7 \times 8.0$ \\
\hline В & 160331.80 & -530921.5 & 9.0 & $3.0 \times 2.5$ \\
\hline С & 160331.91 & -530931.1 & 3.0 & $6.1 \times 2.6$ \\
\hline
\end{tabular}

Note.- Units of right ascension are hours, minutes, and seconds, and units of declination are degrees, arcminutes, and arcseconds.

Walsh et al. (1998). The less compact source (object A) appears to have an irregular shell-like structure.

No radio continuum emission was detected toward IRAS 16272-4837 within a region of $3^{\prime}$ in radius centered on the IRAS position. We set a new $3 \sigma$ upper limit for the $6 \mathrm{~cm}$ flux density of IRAS $16272-4837$ of $0.2 \mathrm{mJy}$, about 30 times smaller than that previously reported by Walsh et al. (1998). We detected, however, emission from a region located about $4^{\prime}$ northwest of the array phase center, with a total flux density (corrected by primary beam) of $122 \mathrm{mJy}$. The morphology of the emission is complex, showing an elongated bipolar-like structure with a peak located at $\alpha=16^{\mathrm{h}} 30^{\mathrm{m}}$ $35^{\mathrm{s}} \mathrm{0}, \delta=-48^{\circ} 40^{\prime} 47^{\prime \prime} .6(\mathrm{~J} 2000.0)$.

\subsection{Millimeter Continuum Emission}

Figure 2 presents maps of the $1.2 \mathrm{~mm}$ continuum emission observed with SEST, revealing the presence of strong emission toward both IRAS sources. The $1.2 \mathrm{~mm}$ emission associated with IRAS $15596-5301$ has a peak position at $\alpha=16^{\mathrm{h}} 03^{\mathrm{m}} 32^{\mathrm{s}} \mathrm{9}, \delta=-53^{\circ} 09^{\prime} 20^{\prime \prime}(\mathrm{J} 2000.0)$, a flux density of $5.8 \pm 0.3 \mathrm{Jy}$, and a peak flux density of $2.0 \mathrm{Jy} \mathrm{beam}^{-1}$. It shows an elongated morphology, with deconvolved major and minor axes of 42 ". 1 and $24 " .6$ at P.A. $=46^{\circ}$. The $1.2 \mathrm{~mm}$ emission detected toward IRAS $16272-4837$ has a peak position at $\alpha=16^{\mathrm{h}} 30^{\mathrm{m}} 58^{\mathrm{s}} 7, \delta=-48^{\circ} 43^{\prime} 55^{\prime \prime}(\mathrm{J} 2000.0)$, a flux density of $13.8 \pm 0.9 \mathrm{Jy}$, and a peak flux density of 4.6 $\mathrm{Jy}_{\text {beam }}{ }^{-1}$. It has an elongated morphology, with deconvolved major and minor axes of $41^{\prime \prime} 0$ and 24.7 at P.A. $=142^{\circ}$.

\subsection{Molecular Line Emission}

The molecular line survey indicates that IRAS 15596-5301 and 16272-4837 have similar chemical characteristics. This is illustrated in Figure 3, which shows rotational diagrams of the emission from selected species $\left(\mathrm{CH}_{3} \mathrm{OH}, \mathrm{CH}_{3} \mathrm{CN}, \mathrm{CH}_{3} \mathrm{CCH}\right.$, and $\left.\mathrm{HCCCN}\right)$ with optically thin lines and at least five observed transitions. The average rotational temperature derived from these diagrams is $27 \mathrm{~K}$ for both sources. Overall, we detected emission from more than 20 species toward both objects. Table 2 summarizes the derived properties of the molecular emission in selected species. Column densities, rotational temperatures, and abundances relative to $\mathrm{CO}$ are given in columns (2)-(4). The column densities of optically thick species, such as $\mathrm{CO}$ and CS, were determined as follows. The optical depths of a pair of an optically thick line and an optically thin line of an isotopic species are determined, for a given abundance ratio, from the ratio of their observed brightness temperatures. From these opacities, and assuming an excitation temperature 


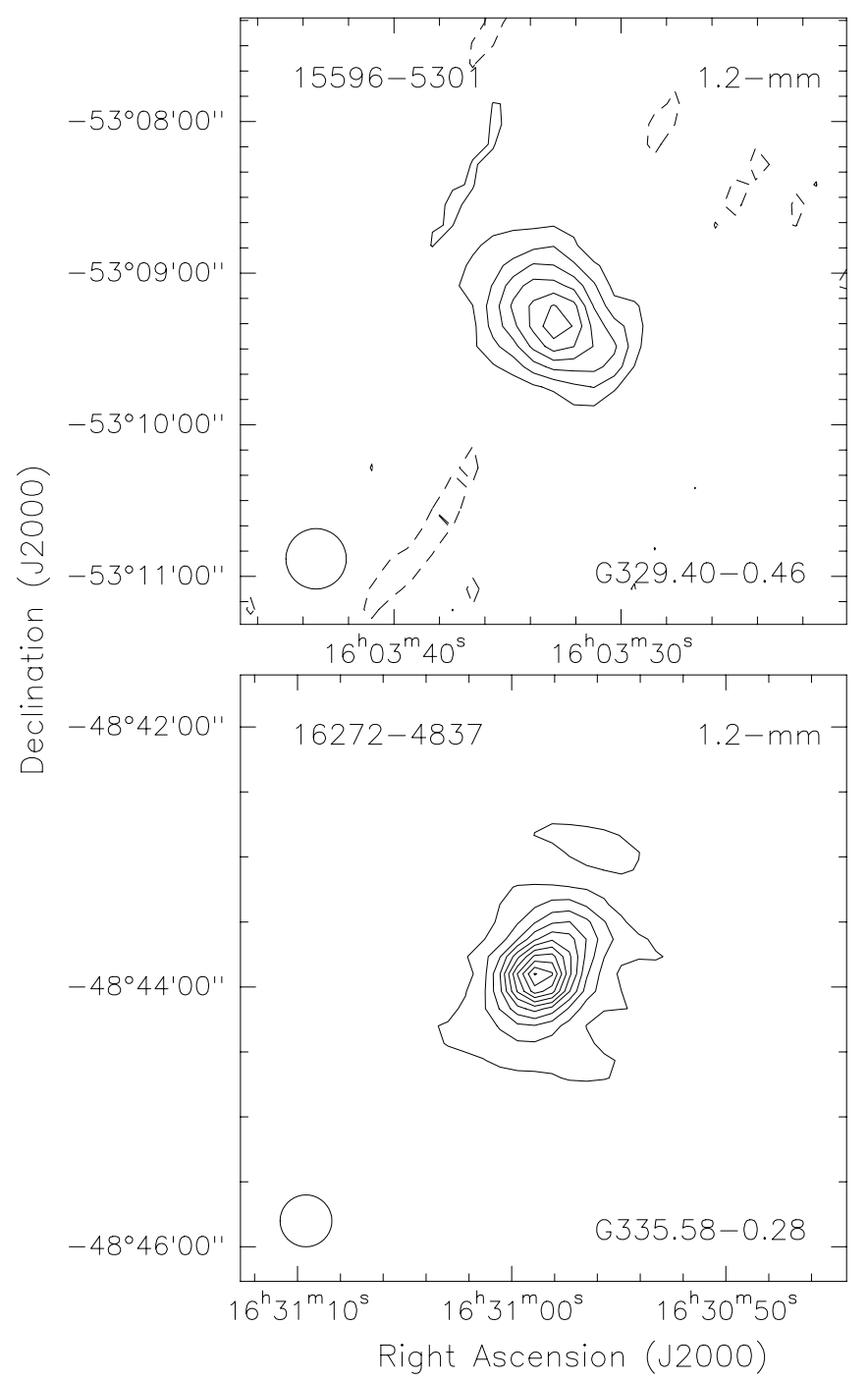

FIG. 2.-Maps of $1.2 \mathrm{~mm}$ continuum emission observed with SEST. The angular resolution is 24" (FWHM beam is shown in the bottom left corner). Top: IRAS 15596-5301. Contour levels are $-1,1,2,3,4,5$, and 6 times 0.3 Jy beam ${ }^{-1}$. Bottom: IRAS 16272-4837. Contour levels are $-1,1,2,3,4,5$, $6,7,8,9$ and 10 times $0.5 \mathrm{Jy}^{\text {beam }}{ }^{-1}$.

of $30 \mathrm{~K}$, we then computed the total column density assuming that energy levels are populated according to local thermodynamic equilibrium (see Bourke et al. 1997 for a description of the method). For the derivation of CO column densities, we used the optically thick ${ }^{13} \mathrm{CO}(1 \rightarrow 0)$ and optically thin $\mathrm{C}^{18} \mathrm{O}(1 \rightarrow 0)$ pair of lines, whereas for $\mathrm{CS}$, we used the $\mathrm{CS}(2 \rightarrow 1)$ and $\mathrm{C}^{34} \mathrm{~S}(2 \rightarrow 1)$ pair of lines. For species with optically thick lines, it is also possible to determine the filling factor of the emission within the observed beam. Filling factors of $\sim 0.5$ for ${ }^{13} \mathrm{CO}$ and $\sim 0.13$ for $\mathrm{CS}$ were estimated for both sources.

Figure 4 shows maps of the $\mathrm{CS}(5 \rightarrow 4)$ line emission from both sources. The morphology of the dense gas from IRAS $15596-5301$ is roughly circular, with a mean angular diameter of $37^{\prime \prime}$. This implies a cloud radius of $0.41 \mathrm{pc}$ at a distance of $4.6 \mathrm{kpc}$. The spatial distribution of the dense gas from IRAS $16272-4837$ is elongated, with major and minor axes of $61^{\prime \prime}$ and $42^{\prime \prime}$, respectively. This implies radii of 0.50 and $0.35 \mathrm{pc}$ at a distance of 3.4 kpc. From the observed CS size and derived CS column density, we estimate that the CS density is $4 \times 10^{-3} \mathrm{~cm}^{-3}$

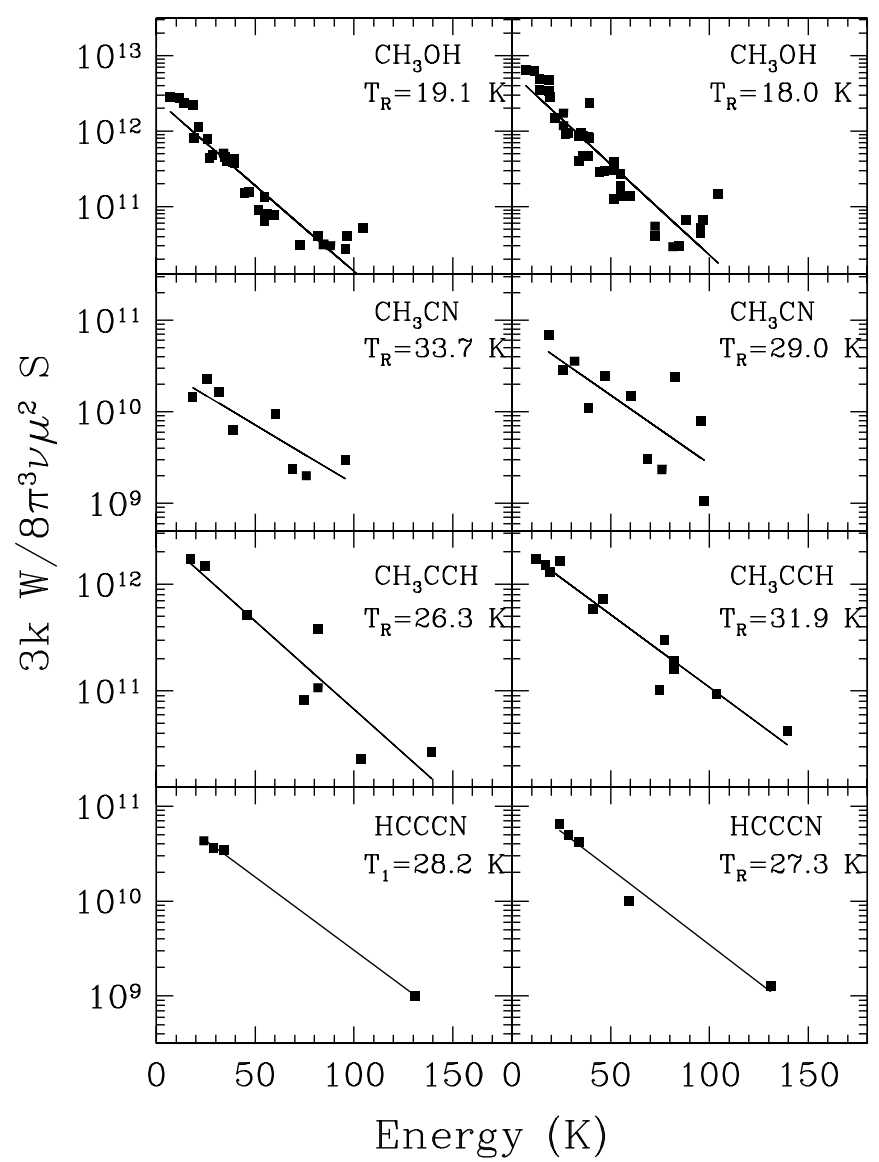

FIG. 3.- Rotational diagrams of the emission from selected species observed toward the IRAS 15596-5301 (left) and 16272-4837 (right) massive dense cores. Top to bottom: $\mathrm{CH}_{3} \mathrm{OH}, \mathrm{CH}_{3} \mathrm{CN}, \mathrm{CH}_{3} \mathrm{CCH}$, and $\mathrm{HCCCN}$. The lines correspond to least-squares linear fits to the observed data. The derived rotational temperatures are given in the top right corners.

in IRAS $15596-5301$ and $2 \times 10^{-3} \mathrm{~cm}^{-3}$ in $16272-4837$. Furthermore, assuming a $\left[\mathrm{CS} / \mathrm{H}_{2}\right]$ abundance ratio of $1 \times 10^{-8}$ (e.g., van der Tak et al. 2000), we find that the molecular hydrogen density and the mass of molecular gas are $4 \times 10^{5} \mathrm{~cm}^{-3}$ and $6.6 \times 10^{3} M_{\odot}$ for IRAS 15596-5301, and $2 \times 10^{5} \mathrm{~cm}^{-3}$ and $2.6 \times 10^{3} M_{\odot}$ for IRAS 16272-4837. Thus, the molecular observations show that both sources are associated with dense, massive molecular cores. The mass of the cores can alternatively be estimated assuming that they are in virial equilibrium (MacLaren, Richardson, \& Wolfendale 1988). From the observed size and average line width in the CS (5-4) line, we derive virial masses of $2.3 \times 10^{3} M_{\odot}$ for IRAS $15596-5301\left(R=0.41 \mathrm{pc} ; \Delta v=5.17 \mathrm{~km} \mathrm{~s}^{-1}\right)$ and $2.6 \times 10^{3} M_{\odot}$ for IRAS $16272-4837(R=0.42 \mathrm{pc}$; $\Delta v=5.44 \mathrm{~km} \mathrm{~s}^{-1}$ ).

Figure 5 shows the spectra observed with the high-resolution spectrometer toward the peak position of the IRAS $16272-4837$ core. The $\mathrm{CS}(2 \rightarrow 1), \mathrm{HCO}^{+}(1 \rightarrow 0)$, and $\mathrm{HCO}^{+}(3 \rightarrow 2)$ spectra show double-peaked line profiles, with a bright blueshifted peak at the velocity of $\sim-47.1 \mathrm{~km}$ $\mathrm{s}^{-1}$ and a weaker redshifted peak at the velocity of $\sim-44.6$ $\mathrm{km} \mathrm{s}^{-1}$. On the other hand, the profiles of the $\mathrm{C}^{34} \mathrm{~S}(2 \rightarrow 1)$ and $\mathrm{H}^{13} \mathrm{CO}^{+}(1 \rightarrow 0)$ lines show a symmetric single component with a peak line center velocity of $\sim-46.0 \mathrm{~km} \mathrm{~s}^{-1}$. These spectroscopic signatures suggest that the bulk of the molecular gas toward IRAS $16272-4837$ is undergoing 
TABLE 2

Molecular Gas Parameters

\begin{tabular}{|c|c|c|c|c|c|c|}
\hline \multirow[b]{2}{*}{$\begin{array}{l}\text { MoleCUle } \\
\text { (1) }\end{array}$} & \multicolumn{3}{|c|}{ IRAS 15596-5301 } & \multicolumn{3}{|c|}{ IRAS $16272-4837$} \\
\hline & $\begin{array}{c}T_{R} \\
(\mathrm{~K}) \\
(2)\end{array}$ & $\begin{array}{c}N \\
\left(\mathrm{~cm}^{-2}\right) \\
(3)\end{array}$ & $\begin{array}{c}{[\mathrm{X} / \mathrm{CO}]} \\
\text { (4) }\end{array}$ & $\begin{array}{c}T_{R} \\
(\mathrm{~K}) \\
(5)\end{array}$ & $\begin{array}{c}N \\
\left(\mathrm{~cm}^{-2}\right) \\
(6)\end{array}$ & $\begin{array}{c}{[\mathrm{X} / \mathrm{CO}]} \\
(7)\end{array}$ \\
\hline $\mathrm{CO} \ldots \ldots$ & $30^{\mathrm{a}}$ & $8.1 \times 10^{19}$ & 1 & $30^{\mathrm{a}}$ & $2.1 \times 10^{19}$ & 1 \\
\hline${ }^{13} \mathrm{CO}$ & $30^{\mathrm{a}}$ & $1.5 \times 10^{18}$ & $1.9 \times 10^{-2}$ & $30^{\mathrm{a}}$ & $3.8 \times 10^{17}$ & $1.8 \times 10^{-2}$ \\
\hline $\mathrm{C}^{18} \mathrm{O} \ldots \ldots \ldots \ldots \ldots$ & $30^{\mathrm{a}}$ & $1.1 \times 10^{17}$ & $1.4 \times 10^{-3}$ & $30^{\mathrm{a}}$ & $2.7 \times 10^{16}$ & $1.3 \times 10^{-3}$ \\
\hline $\mathrm{CS} \ldots \ldots \ldots \ldots \ldots \ldots$ & $30^{\mathrm{a}}$ & $9.8 \times 10^{15}$ & $1.2 \times 10^{-4}$ & $30^{\mathrm{a}}$ & $4.2 \times 10^{15}$ & $2.0 \times 10^{-4}$ \\
\hline$C^{34} \mathrm{~S} \ldots \ldots$ & $30^{\mathrm{a}}$ & $4.4 \times 10^{14}$ & $5.4 \times 10^{-6}$ & $30^{\mathrm{a}}$ & $1.9 \times 10^{14}$ & $9.0 \times 10^{-6}$ \\
\hline $\mathrm{H}_{2} \mathrm{CO} \ldots \ldots$ & & & & 27.0 & $9.4 \times 10^{13}$ & $4.5 \times 10^{-6}$ \\
\hline $\mathrm{CH}_{3} \mathrm{OH} \ldots \ldots \ldots \ldots$ & 19.1 & $2.7 \times 10^{14}$ & $3.3 \times 10^{-6}$ & 18.0 & $5.5 \times 10^{14}$ & $2.6 \times 10^{-5}$ \\
\hline $\mathrm{CH}_{3} \mathrm{CCH} \ldots \ldots .$. & 26.3 & $2.1 \times 10^{14}$ & $2.6 \times 10^{-6}$ & 31.9 & $2.3 \times 10^{14}$ & $1.1 \times 10^{-5}$ \\
\hline $\mathrm{CH}_{3} \mathrm{CN} \ldots \ldots \ldots$ & 33.7 & $9.0 \times 10^{12}$ & $1.1 \times 10^{-7}$ & 29.0 & $1.9 \times 10^{13}$ & $9.0 \times 10^{-7}$ \\
\hline $\mathrm{CH}_{3} \mathrm{CHO} \ldots \ldots \ldots$ & 25.0 & $7.2 \times 10^{12}$ & $8.9 \times 10^{-8}$ & 21.0 & $1.3 \times 10^{13}$ & $6.2 \times 10^{-7}$ \\
\hline $\mathrm{SiO}$ & 10.4 & $2.9 \times 10^{12}$ & $3.6 \times 10^{-8}$ & 9.9 & $1.0 \times 10^{13}$ & $4.8 \times 10^{-7}$ \\
\hline 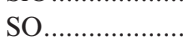 & 23.0 & $6.3 \times 10^{13}$ & $7.8 \times 10^{-7}$ & 11.7 & $8.5 \times 10^{13}$ & $4.0 \times 10^{-6}$ \\
\hline 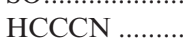 & 28.2 & $4.1 \times 10^{13}$ & $5.1 \times 10^{-7}$ & 27.3 & $5.1 \times 10^{13}$ & $2.4 \times 10^{-6}$ \\
\hline 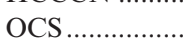 & 37.5 & $3.9 \times 10^{13}$ & $4.8 \times 10^{-7}$ & 28.5 & $1.3 \times 10^{14}$ & $6.2 \times 10^{-6}$ \\
\hline $\mathrm{SO}_{2} \ldots \ldots \ldots \ldots \ldots$ & 27.6 & $1.9 \times 10^{13}$ & $2.3 \times 10^{-7}$ & 25.9 & $1.6 \times 10^{13}$ & $7.6 \times 10^{-7}$ \\
\hline
\end{tabular}

a Assumed excitation temperature.

large-scale inward motion. Infalling motion traced by optically thick molecular lines is expected to produce line profiles showing blue asymmetry, whereas optically thin lines are expected to exhibit symmetrical profiles (Mardones et al. 1997).

In some species, particularly $\mathrm{SiO}$ and $\mathrm{SO}$, the spectra show the presence of strong wing emission, indicative of outflow activity. In the $\mathrm{SiO}(2 \rightarrow 1)$ line, the wing emission extends up to velocities of -19.6 and $19.5 \mathrm{~km} \mathrm{~s}^{-1}$ relative to the ambient cloud velocity of $-46.2 \mathrm{~km} \mathrm{~s}^{-1}$. Figure 6 shows contour maps of the velocity-integrated $\mathrm{SiO}$ emission in the range of flow velocities from -19.5 to $-3.8 \mathrm{~km} \mathrm{~s}^{-1}$ (blueshifted gas: solid lines) and from 4.2 to $15.9 \mathrm{~km} \mathrm{~s}^{-1}$ (redshifted gas: dashed lines). The flow velocity is defined as $v_{\text {flow }}=v_{\text {LSR }}-v_{0}$, where $v_{0}$ is the velocity of the emission from quiescent ambient gas. The spatial distribution of the wing emission clearly shows a bipolar structure with redshifted and blueshifted lobes extending in opposite directions of a $22 \mu \mathrm{m}$ source detected by the Midcourse Space Experiment (MSX; see later discussion). The bipolar structure shows a moderate degree of collimation, with the blueshifted emission seen mainly toward the west and the redshifted emission seen mainly toward the east, suggesting that the underlying wind is a wide-angle wind.

\subsection{Mid-Infrared Emission}

We have used the database from the MSX (Price 1995) survey of the Galactic plane (Egan et al. 1998) to investigate the mid-infrared emission in the four $M S X$ broad bands ( $A$ : 6.8-10.8 $\mu \mathrm{m}, C: 11.1-13.2 \mu \mathrm{m}, D: 13.5-15.9 \mu \mathrm{m}$, and $E$ : $18.2-25.1 \mu \mathrm{m})$ from both IRAS sources. Figure 7 presents gray-scale images of the emission in the $A, C$, and $E$ bands of a region of about $4^{\prime}$ toward IRAS 15596-5301. These images show the presence of two sources within the central $1^{\prime}$ region: a compact object, brighter in the $E$-band image, and an extended object, brighter in the $C$ band, with a peak located about $30^{\prime \prime}$ southwest of the former. Both sources are seen in emission in the four $M S X$ bands. The three radio continuum sources detected within this region (delineated by the $0.5 \mathrm{mJy}$ contour level in the top panel of Fig. 7), as well as the peak position of IRAS 15596-5301 (marked by the cross), lie projected within the face of the compact object. Furthermore, the extent of the $1.2 \mathrm{~mm}$ dust continuum emission (delineated by the $0.5 \mathrm{Jy}$ contour level in the bottom panel of Fig. 7) is similar to that of the $E$-band infrared emission. These results show that the compact $M S X$ object is intimately associated with the IRAS 15596-5301 massive molecular core. We measured flux densities of 5.4, 4.1 , and $24.3 \mathrm{Jy}$ in the $C, D$, and $E$ bands, respectively. The extended object shows strong emission in the $A$ and $C$ bands, which are sensitive to emission from polycyclic aromatic hydrocarbon features (7.7 and $8.3 \mu \mathrm{m}$ in the $A$ band; 11.3 and $12.6 \mu \mathrm{m}$ in the $C$ band). Since no radio emission is detected toward this $M S X$ source, we suggest that it might correspond to a photodissociated region excited by a nonionizing star.

IRAS $16272-4837$ is associated with an infrared dark cloud seen in absorption against bright mid-infrared emission in the $A, C$, and $D$ bands. This is shown in the top two panels of Figure 8, which present gray-scale images of the emission in the $A, C$, and $E$ bands toward IRAS $16272-4837$. There is a good agreement between the molecular, millimeter, and mid-infrared morphologies, as illustrated by the contour of the $1.2 \mathrm{~mm}$ continuum emission shown in Figure 8. From the molecular hydrogen column density derived from the line observations (of $3.4 \times 10^{23}$ $\mathrm{cm}^{-2}$ ), assuming a gas-to-dust mass ratio of 110 (Draine \& Lee 1984) and a dust opacity at $8 \mu \mathrm{m}$ of $10^{3} \mathrm{~cm}^{2} \mathrm{~g}^{-1}$ (Ossenkopf \& Henning 1994), we estimate that the optical depth at $8 \mu \mathrm{m}$ is $\sim 12$, indicating that this cloud is optically thick at mid-infrared. Furthermore, the $M S X$ observations in the $E$ band show the presence, near the center of the infrared dark cloud, of an object seen in emission located at $\alpha=16^{\mathrm{h}} 30^{\mathrm{m}}$ $58^{\mathrm{s}} .18, \delta=-48^{\circ} 43^{\prime} 47^{\prime \prime} .9$ (J2000.0) (see bottom panel of Fig. 8). We finally note that the $M S X$ object seen near the top right corner of Figure 8, which is clearly extended in the $A$-band image, is associated with IRAS 16269-4834 and coincident with the radio source detected $\sim 4^{\prime}$ northwest of IRAS $16272-4837$. 


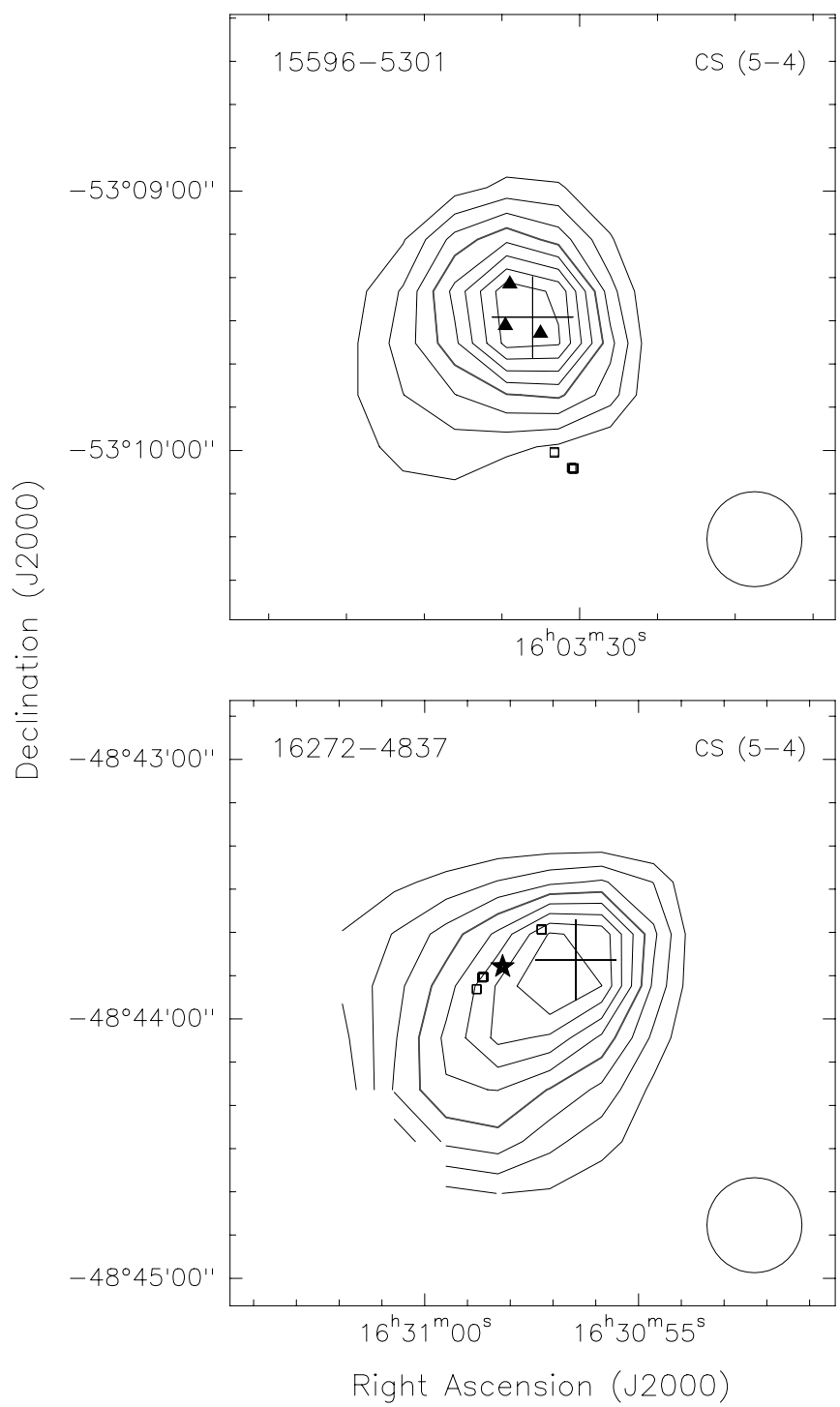

FIG. 4.-Maps of CS(5 $\rightarrow 4)$ line emission observed with SEST. The angular resolution is 22" (FWHM beam is shown in the bottom right corner). Top: IRAS 15596-5301. Contour levels are 20, 30, 40, 50, 60, 70, 80 , and $90 \%$ of the peak velocity-integrated antenna temperature of $11.4 \mathrm{~K}$ $\mathrm{km} \mathrm{s}^{-1}$. The triangles mark the positions of the compact $\mathrm{H}$ II regions. Bottom: IRAS 16272-4837. Contour levels are 20, 30, 40, 50, 60, 70, 80, and $90 \%$ of the peak velocity-integrated antenna temperature of $7.2 \mathrm{~K} \mathrm{~km} \mathrm{~s}^{-1}$. The star marks the peak position of the $22 \mu \mathrm{m} \mathrm{MSX}$ source. In both panels, the cross marks the position of the IRAS source and the squares the position of $6.67 \mathrm{GHz}$ methanol masers (Walsh et al. 1998).

\section{DISCUSSION}

\subsection{Spectral Energy Distribution}

Figure 9 shows the spectral energy distribution (SED) of IRAS $15596-5301$ and $16272-4837$ from $12 \mu \mathrm{m}$ to $1.2 \mathrm{~mm}$, which is mainly due to thermal dust emission. We fitted the SED with modified blackbody functions of the form $B_{\nu}\left(T_{d}\right)\left[1-\exp \left(-\tau_{\nu}\right)\right] \Omega_{S}$, where $\tau_{\nu}$ is the dust optical depth, $B_{\nu}\left(T_{d}\right)$ is the Planck function at the dust temperature $T_{d}$, and $\Omega_{s}$ is the solid angle subtended by the dust-emitting region. The opacity was assumed to vary with frequency as $\nu^{\beta}$, i.e., $\tau_{\nu}=\left(\nu / \nu_{0}\right)^{\beta}$, where $\nu_{0}$ is the frequency at which the optical depth is unity. Due to the limited number of spectral points, we have set the value of $\beta$ equal to 2.0 , consistent with tabulated opacities (Ossenkopf \& Henning 1994) and derived values for high-mass star-forming regions (Molinari et al. 2000). A single-temperature model produced poor fits, underestimating the emission observed at wavelengths smaller than $25 \mu \mathrm{m}$, and therefore we used a model with two temperature components.

From the fits (long-dashed lines), we derive that the colder dust components (short-dashed lines) toward IRAS 15596-5301 and 16272-4837 have, respectively, temperatures of 27 and $25 \mathrm{~K}$, angular sizes (assuming a Gaussian flux distribution) of $30^{\prime \prime}$ (FWHM), and wavelengths at which the opacity is unity of $\sim 90$ and $140 \mu \mathrm{m}$. The temperature of the hot-dust component is $100 \mathrm{~K}$ for IRAS $15596-5301$ and $115 \mathrm{~K}$ for IRAS $16272-4837$. The thermal dust emission at $1.2 \mathrm{~mm}$ is therefore optically thin $\left(\tau_{1.2 \mathrm{~mm}} \sim 5 \times 10^{-3}\right)$, and thus the observed flux density at $1.2 \mathrm{~mm}$ allows us to obtain an additional mass estimate of the dense cores. In general, for an isothermal dust source, the total gas mass $M_{g}$ is given in terms of the observed flux density $S_{\nu}$ at an optically thin frequency $\nu$ by (e.g., Chini, Krügel, \& Wargau 1987)

$$
M_{g}=\frac{S_{\nu} D^{2}}{R_{\mathrm{dg}} \kappa_{\nu} B_{\nu}\left(T_{d}\right)},
$$

where $\kappa_{\nu}$ is the mass absorption coefficient of the dust, $R_{\mathrm{dg}}$ is the dust-to-gas mass ratio (assuming 10\% $\mathrm{He}$ ), and $B_{\nu}\left(T_{d}\right)$ is the Planck function at the dust temperature $T_{d}$. The main source of uncertainty in the conversion of the observed flux density into gas mass is the $R_{\mathrm{dg}} \kappa_{\nu}$ factor, or total mass opacity, which is a poorly known quantity (e.g., Gordon 1995). Using a dust opacity at $1.2 \mathrm{~mm}$ of $1 \mathrm{~cm}^{2} \mathrm{~g}^{-1}$, as computed by Ossenkopf \& Henning (1994) for dense and cold protostellar cores, $R_{\mathrm{dg}}=0.01$, the fitted dust temperatures, and the observed flux densities, we derive masses of $1.4 \times 10^{3}$ $M_{\odot}$ for IRAS $15596-5301$ and $2.0 \times 10^{3} M_{\odot}$ for IRAS 16272-4837. These masses derived from the dust emission are in good agreement with those derived from the molecular line intensities and from the virial assumption.

\subsection{Evolutionary Stages \\ 4.2.1. IRAS 15596-5301 (G329.40-0.46)}

The radio continuum observations toward IRAS $15596-5301$ indicate the presence, in a region of $\sim 0.3 \mathrm{pc}$ in radius, of three distinct $\mathrm{H}$ II regions with diameters ranging from 0.06 to $0.2 \mathrm{pc}$. The multiple structure of the ionized gas is typical of Galactic $\mathrm{H}$ II regions and is most likely due to the presence of a cluster of exciting stars. If components $\mathrm{A}, \mathrm{B}$, and $\mathrm{C}$ are excited by individual zeroage main-sequence stars, the rates of UV photons needed to ionize them (see Table 3) imply exciting stars with spectral types of B0, B0.5, and B1, respectively. The total luminosity emitted by this cluster of B stars, as inferred from the radio observations, is $4.1 \times 10^{4} L_{\odot}$. On the other hand, the total far-infrared luminosity computed using the IRAS fluxes (see Casoli et al. 1986) is $\sim 6.5 \times 10^{4} L_{\odot}$ (assuming a distance of $4.6 \mathrm{kpc}$; L. Bronfman 2002, private communication). The difference between the radio-derived luminosity and the IRAS luminosity could be explained by the presence of dust within the H II regions. Garay et al. (1993) found that the fraction of Lyman continuum photons absorbed by dust within $\mathrm{H}$ II regions is typically $55 \%$. Alternatively, it could be explained by the presence, in addition to the B 

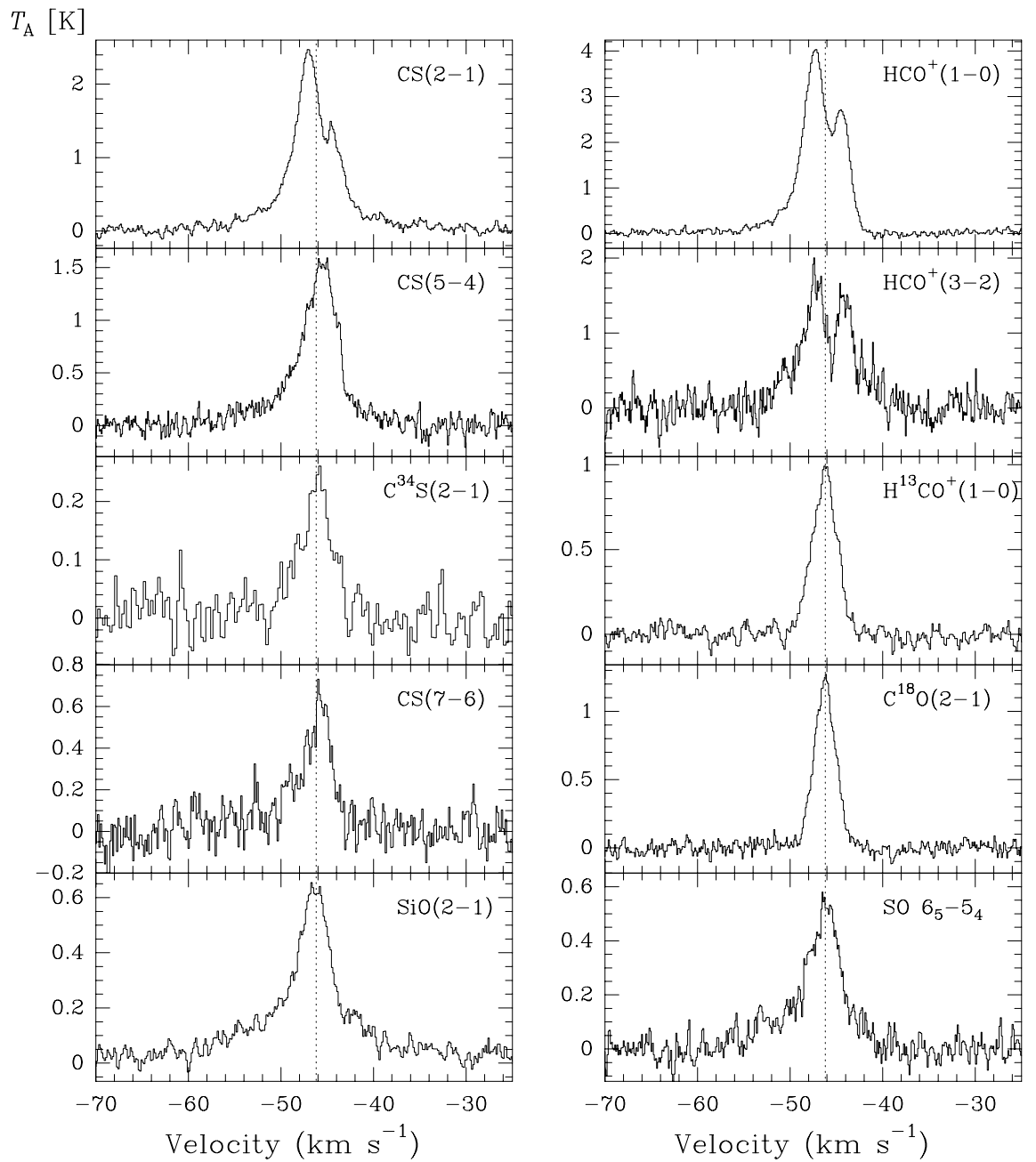

FIG. 5.- Spectra of the emission in several molecular lines observed toward the peak position of the IRAS $16272-4837$ massive core. Transitions are given in the top right corner of the spectra. The vertical dotted line indicates the systemic velocity of the ambient gas of $-46.2 \mathrm{~km} \mathrm{~s}^{-1}$.

stars, of several less massive stars that contribute to the far-infrared luminosity but that are not hot enough to contribute to ionization. It is not easy, however, to disentangle which of these effects play the predominant role.

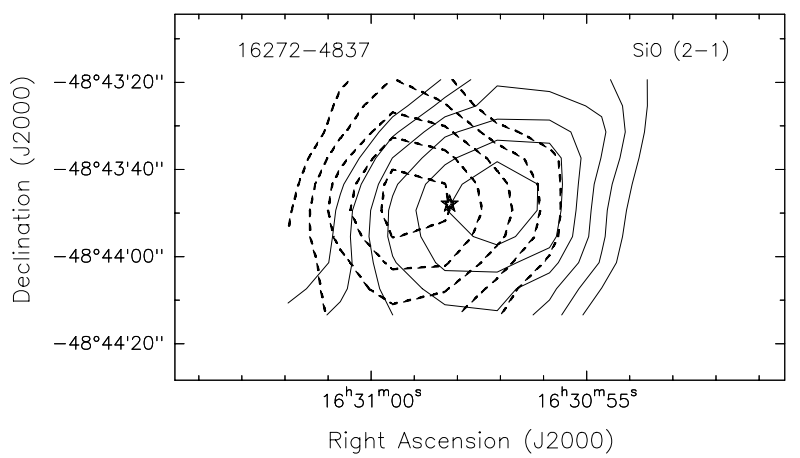

FIG. 6.-Contour maps of velocity-integrated $\mathrm{SiO}(2 \rightarrow 1)$ line wing emission from IRAS 16272-4837. Solid lines represent blueshifted emission $\left(v_{\text {flow }}=-19.5\right.$ to $\left.-3.8 \mathrm{~km} \mathrm{~s}^{-1}\right)$ and dashed lines, redshifted emission $\left(v_{\text {flow }}=4.2\right.$ to $\left.15.9 \mathrm{~km} \mathrm{~s}^{-1}\right)$. The star marks the position of the $22 \mu \mathrm{m} M S X$ source. Contour levels are at $0.60,0.72,0.84,0.96,1.08$, and $1.20 \mathrm{~K} \mathrm{~km} \mathrm{~s}^{-1}$.
The compact $\mathrm{H}$ II regions are found projected toward the peak of the CS( $5 \rightarrow 4)$ emission map (see Fig. 4), suggesting that they are deeply embedded within the dense molecular core. From the observed sizes, and assuming a sound speed in the ionized gas of $11.4 \mathrm{~km} \mathrm{~s}^{-1}$, we estimate that the $\mathrm{H}$ II regions have dynamical ages between $3 \times 10^{3}$ and $8 \times 10^{3} \mathrm{yr}$. If these correspond to the actual ages of the compact $\mathrm{H}$ II regions, then we should conclude that they are very young objects. The dynamical timescales, however, may not provide a realistic estimate of the actual age of the $\mathrm{H}$ II regions. The large number of $\mathrm{UC} \mathrm{H}$ II regions and their short dynamical ages poses the well-known problem that the rate of massive star formation appears to be much greater than other indicators suggest (Wood \& Churchwell 1989; Churchwell 1990). Due to the high density of the molecular gas in which they are embedded, we suggest instead that the $\mathrm{H}$ II regions within the IRAS 15596-5301 massive core might be in pressure equilibrium with the surrounding dense ambient medium and are currently stalled at their equilibrium radius (e.g., De Pree, Rodríguez, \& Goss 1995). The molecular density of the ambient gas needed to stall an $\mathrm{H}$ II region at radius $R_{f}$ is given by (e.g., Garay \& 

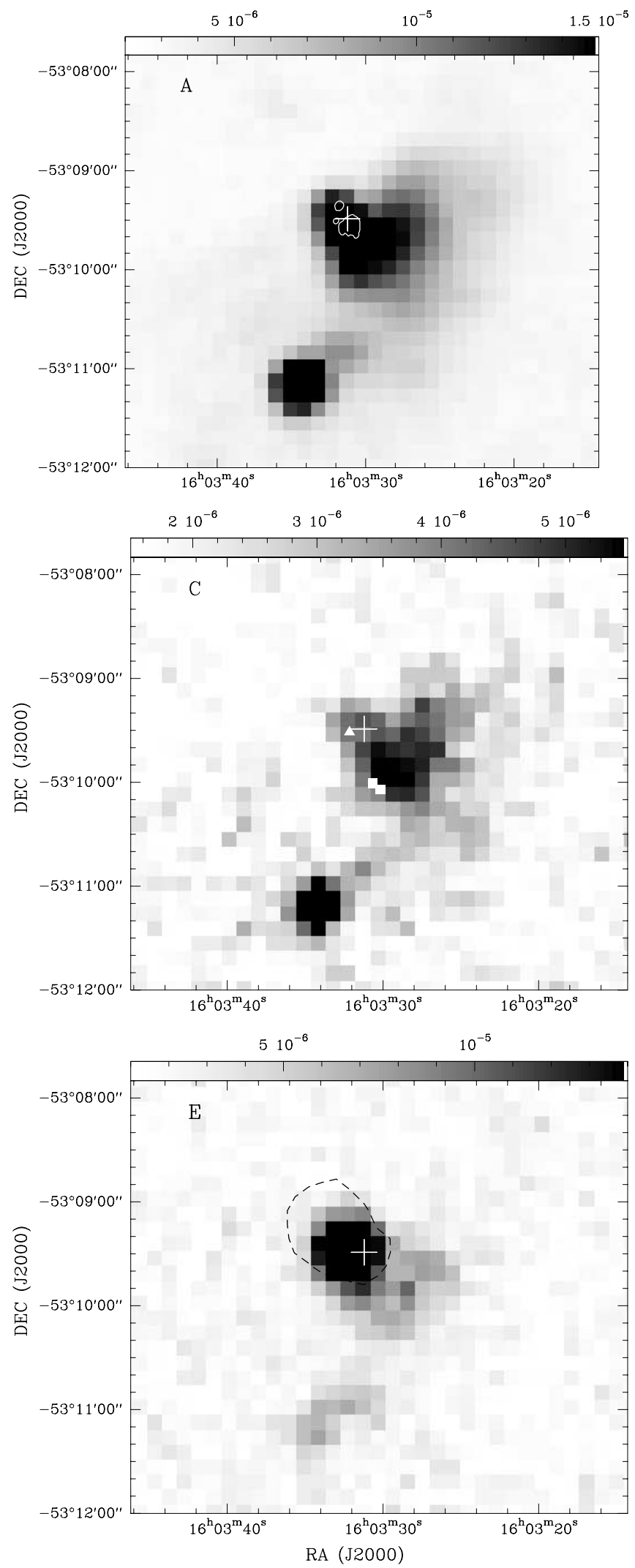

FIG. 7.-MSX images (gray-scale) toward IRAS 15596-5301. The cross marks the peak position of the IRAS source. The intensity scale is in units of $\mathrm{W} \mathrm{m}^{-2} \mathrm{sr}^{-1}$. Top: $A$-band image. The contour delineates the radio continuum emission at the level of $0.5 \mathrm{mJy}$. Middle: $C$-band image. The squares and triangles mark the position of $6.67 \mathrm{GHz}$ methanol and hydroxyl masers, respectively. Bottom: E-band image. The dotted contour indicates the $1.2 \mathrm{~mm}$ dust emission at the level of $0.5 \mathrm{Jy}$.
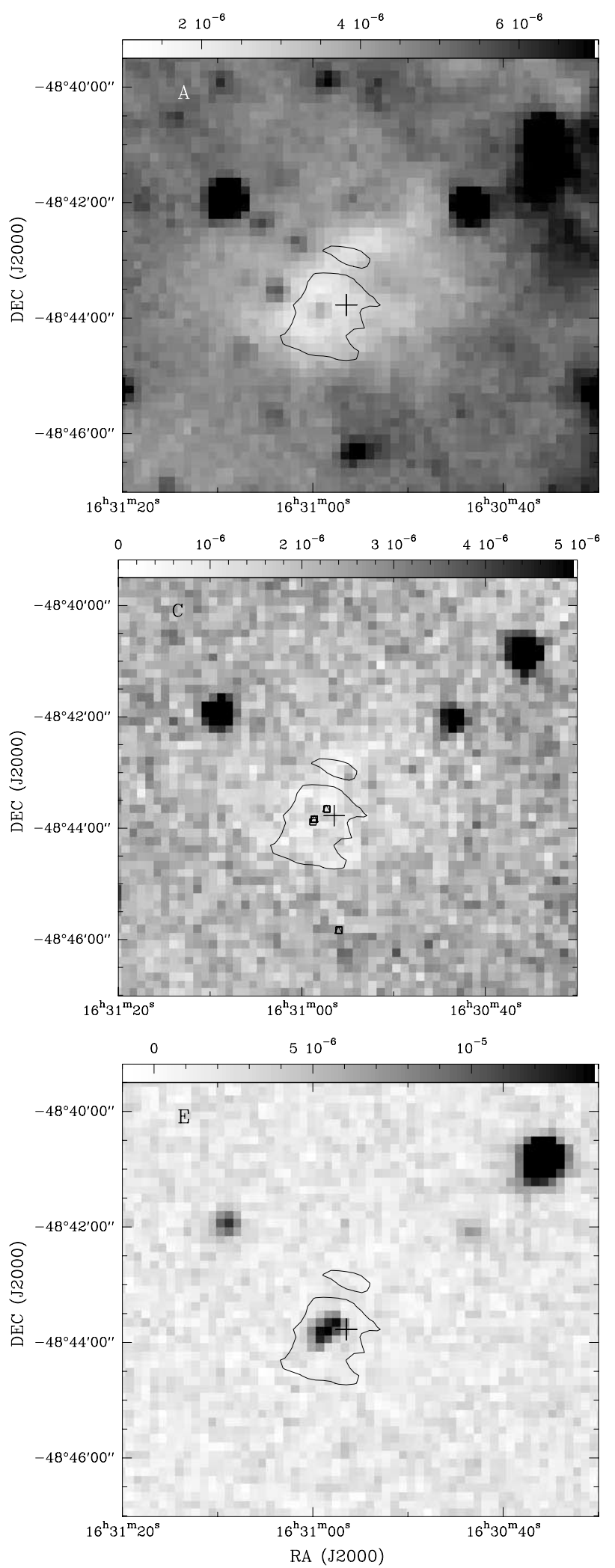

FIG. 8. - MSX images (gray-scale) toward IRAS 16272-4837. The cross marks the peak position of the IRAS source and the contour delineates the $1.2 \mathrm{~mm}$ continuum emission at the level of $0.5 \mathrm{Jy}$. The intensity scale is in units of $\mathrm{W} \mathrm{m}^{-2} \mathrm{sr}^{-1}$. Top: $A$-band image. The light areas in the gray scale correspond to regions of lower emission than the bright Galactic plane emission and indicate the infrared extinction cloud associated with IRAS 16272-4837. Middle: $C$-band image. The squares and triangles mark the position of $6.67 \mathrm{GHz}$ methanol and hydroxyl masers, respectively. Bottom: E-band image. 
TABLE 3

Derived Parameters of H it Regions

\begin{tabular}{|c|c|c|c|c|c|}
\hline H II Region & $\begin{array}{l}\text { Diameter } \\
\text { (pc) }\end{array}$ & $\begin{array}{c}\text { EM } \\
\left(\mathrm{pc} \mathrm{cm}^{-6}\right)\end{array}$ & $\begin{array}{c}n_{e} \\
\left(\mathrm{~cm}^{-3}\right)\end{array}$ & $\begin{array}{c}N_{u} \\
\left(\mathrm{~s}^{-1}\right)\end{array}$ & Spectral Type \\
\hline & 0.196 & $2.4 \times 10^{5}$ & $9.2 \times 10^{2}$ & $8.3 \times 10^{46}$ & $\mathrm{~B} 0$ \\
\hline В $\ldots \ldots \ldots \ldots \ldots$ & 0.061 & $5.2 \times 10^{5}$ & $2.4 \times 10^{3}$ & $1.7 \times 10^{46}$ & B0.5 \\
\hline $\mathrm{C} \ldots \ldots \ldots \ldots \ldots$ & 0.089 & $8.0 \times 10^{4}$ & $7.8 \times 10^{2}$ & $5.7 \times 10^{45}$ & B1 \\
\hline
\end{tabular}

Lizano 1999)

$$
\begin{aligned}
\left(\frac{n_{\mathrm{H}_{2}}}{10^{5} \mathrm{~cm}^{-3}}\right)= & 1.9\left(\frac{N_{u}}{10^{49} \mathrm{~s}^{-1}}\right)^{1 / 2}\left(\frac{T_{e}}{10^{4} \mathrm{~K}}\right) \\
& \times\left(\frac{30 \mathrm{~K}}{T_{0}}\right)\left(\frac{1 \mathrm{pc}}{R_{f}}\right)^{3 / 2},
\end{aligned}
$$

where $N_{u}$ is the rate of ionizing UV photons emitted by the exciting star, $T_{e}$ is the electron temperature of the ionized gas, and $T_{0}$ is the temperature of the ambient gas. Using the observed radius and the derived ionizing rate of UV photons of the $\mathrm{H}$ II regions within IRAS 15596-5301, we find that molecular densities of $\sim 5 \times 10^{5}$ $\mathrm{cm}^{-3}$ are needed for them to be pressure confined by the

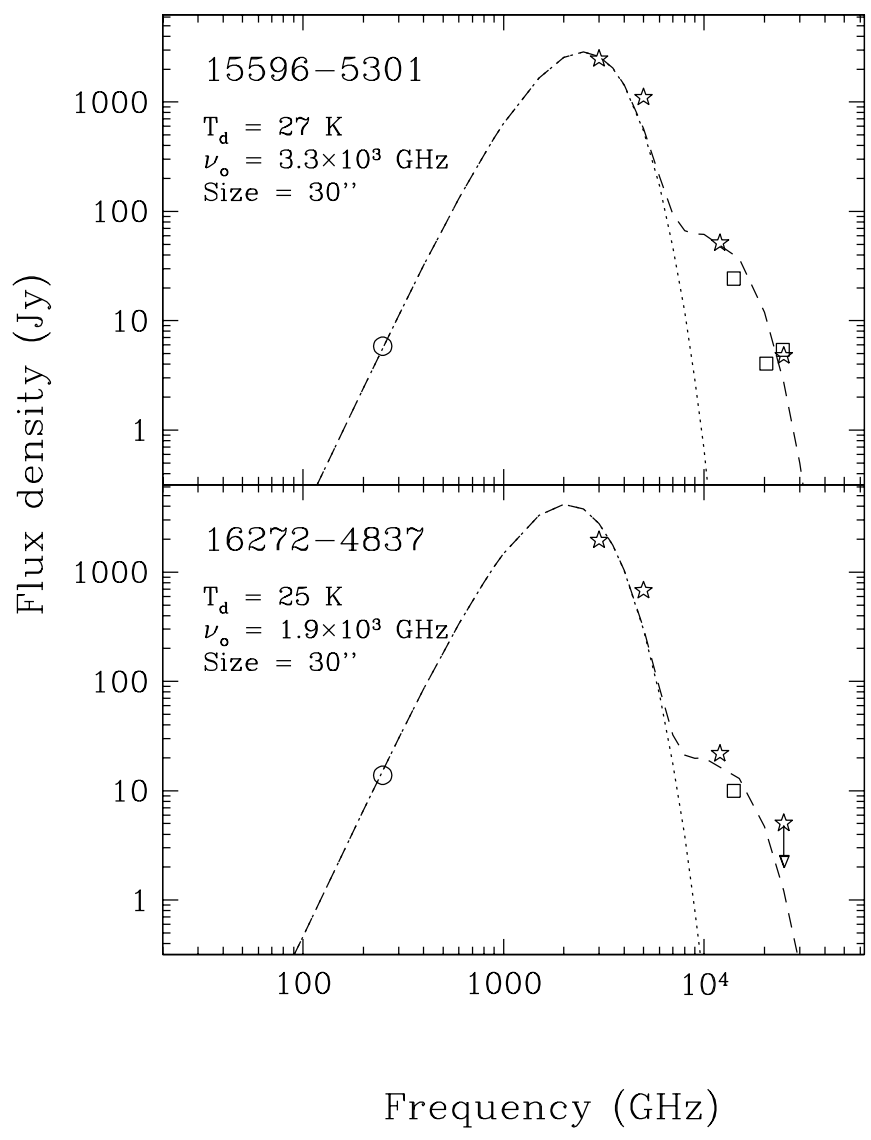

Fig. 9.-Spectral energy distributions. Stars mark IRAS fluxes, squares, $M S X$ fluxes, and the circle, the SIMBA flux. The long-dashed curve is a fit to the spectrum using two modified blackbody functions of the form $B_{\nu}\left(T_{d}\right)\left\{1-\exp \left[-\left(\nu / \nu_{0}\right)^{\beta}\right]\right\}$, with different temperatures. The shortdashed line indicates the fit for the colder temperature component (fit parameters indicated on the top left). Top: IRAS 15596-5301. Bottom: IRAS $16272-4837$ (12 $\mu \mathrm{m}$ flux is an upper limit). dense environment. These densities are similar to those derived from the molecular observations. The time needed for the $\mathrm{H}$ II regions to achieve pressure equilibrium is between $1.0 \times 10^{5}$ and $2.5 \times 10^{5} \mathrm{yr}$, implying that massive star formation started within this core more than $2.5 \times 10^{5} \mathrm{yr}$ ago. We conclude that the dense massive core is in an advanced stage of early evolution, in which multiple OB star formation has already taken place near its central region.

\subsubsection{IRAS $16272-4837(G 335.58-0.28)$}

The total far-infrared luminosity of IRAS 16272-4837 computed using the IRAS fluxes (see Casoli et al. 1986) is $\sim 2.4 \times 10^{4} L_{\odot}$ (assuming a distance of $3.4 \mathrm{kpc}$; L. Bronfman 2002, private communication). The luminosity obtained integrating under the fitted curve in Figure 9 is similar to the IRAS luminosity. The high luminosity suggests that the IRAS 16272-4837 massive core hosts a young massive protostar inside, and the lack of radio emission suggests that it is still undergoing an intense accretion phase. Models of massive envelopes accreting onto a young massive central B-type star (e.g., Osorio et al. 1999) require accretion rates in the envelopes of $\dot{M}>5 \times 10^{-4} M_{\odot} \mathrm{yr}^{-1}$ in order to fit the observed SEDs. The high mass accretion rate of the infalling material quenches the development of a UC H II region (Yorke 1984), and the free-free emission from the ionized material is undetectable at centimeter wavelengths.

This hypothesis is supported by the characteristics of the line profiles observed toward IRAS 16272-4837, which suggest that the molecular gas is undergoing infalling motions. From the spectra of the optically thick $\mathrm{HCO}^{+}(1 \rightarrow 0)$ line, we measure a velocity difference between the red and blue peaks of $2.7 \mathrm{~km} \mathrm{~s}^{-1}$ and brightness temperatures of the blue peak, red peak, and dip of 5.4, 3.6, and $2.9 \mathrm{~K}$, respectively. From the spectra of the optically thin $\mathrm{H}^{13} \mathrm{CO}^{+}(1 \rightarrow 0)$ line, we measure a FWHM line width of $3.18 \mathrm{~km} \mathrm{~s}^{-1}$. From these values, using the simple model of contracting clouds of Myers et al. (1996), we derive a characteristic inward speed of $0.5 \mathrm{~km} \mathrm{~s}^{-1}$. We note that this value is considerably smaller than the free-fall velocity expected for a cloud with a total mass of $\sim 3 \times 10^{3} M_{\odot}$ at its outer envelope radius of $0.4 \mathrm{pc}$, suggesting that the collapse is not dynamical. Using the derived values of the infall speed, molecular density, and core size, we obtain a mass infall rate $\dot{M}_{\text {in }}$ of $1 \times 10^{-2} M_{\odot} \mathrm{yr}^{-1}$, large enough to prevent the development of a $\mathrm{UC} \mathrm{H}$ II region. The high value of the mass infall rate raises the question of what fraction of the total luminosity is due to accretion. The accretion luminosity $L_{\text {acc }}$ is

$$
L_{\mathrm{acc}}=\frac{G f \dot{M}_{\mathrm{in}} M_{p}}{R_{p}},
$$


where $f$ is the fraction of the large-scale mass infall rate that goes into accretion onto the protostar, $M_{p}$ is the mass of the protostar, and $R_{p}$ the radius at which the shock occurs. None of these three parameters are known for IRAS 16272-4837. Assuming $M_{p} \sim 10 M_{\odot}, R_{p} \sim 3 \times 10^{12} \mathrm{~cm}$, and $f \sim 0.05$ (e.g., Norberg \& Maeder 2000), we obtain $L_{\text {acc }} \sim 3.6 \times 10^{3} L_{\odot}$, about $15 \%$ of the total luminosity. We emphasize that this value of the accretion luminosity only corresponds to a rough estimate, particularly because the value of $f$ is highly uncertain.

Additional evidence for IRAS $16272-4837$ to be in a collapsing stage is provided by the presence of bipolar outflowing gas, a phenomenon that is thought to be closely related to accretion processes. The $22 \mu \mathrm{m}$ object lies at the center of symmetry of the $\mathrm{SiO}$ outflow, suggesting that it is intimately associated with the energy source of the outflow. The early evolutionary stage of this region is also indicated by the presence of $6.67 \mathrm{GHz}$ methanol masers, which are thought to be signposts of young regions of massive star formation (Walsh et al. 1997, 1998). Note that the $22 \mu \mathrm{m}$ source is elongated, with the $6.67 \mathrm{GHz}$ methanol masers being aligned along its major axis. Finally, we mention that the high value of the mass-to-luminosity ratio of IRAS $16272-4837, M / L=0.083$, about 4 times higher than that of IRAS 15596-5301, is another indicator of its youth, as argued by Sridharan et al. (2002).

\section{SUMMARY}

We undertook sensitive radio continuum observations at $4.8 \mathrm{GHz}$ using ATCA and 1.2 millimeter continuum and molecular line observations using SEST toward IRAS 15596-5301 and 16272-4837, two luminous objects $\left(L>2 \times 10^{4} L_{\odot}\right)$ thought to represent massive star-forming regions in early stages of evolution. The main results and conclusions are summarized as follows:

1. The $4.8 \mathrm{GHz}$ radio continuum observations toward IRAS 15596-5301 show the presence of three distinct compact sources with diameters of $0.06-0.2 \mathrm{pc}$, all located within a region of $30^{\prime \prime}$ in diameter. If they are regions of ionized gas, they have electron densities of $8 \times 10^{2}-2 \times 10^{3}$ $\mathrm{cm}^{-3}$ and are excited by early B-type stars. The $1.2 \mathrm{~mm}$ continuum observations show that the dust emission arises from an elongated region with major and minor axes of 42 ". 1 and 24".6, respectively. The observed $1.2 \mathrm{~mm}$ flux density of $5.8 \mathrm{Jy}$ implies a mass of $1.4 \times 10^{3} M_{\odot}$. The line observations indicate a region of molecular gas with a radius of $\sim 0.4 \mathrm{pc}$, a molecular hydrogen density of $4 \times 10^{5} \mathrm{~cm}^{-3}$, and a rotational temperature of $\sim 27 \mathrm{~K}$. We conclude that IRAS 15596-5301 corresponds to a dense massive $\left(\sim 3 \times 10^{3} M_{\odot}\right)$ molecular core, with a radius of about $0.4 \mathrm{pc}$, that is in the compact $\mathrm{H}$ II region phase of evolution, already hosting a cluster of massive B-type stars. Furthermore, we suggest that the regions of ionized gas excited by these stars are in pressure equilibrium with the ambient molecular gas.

2. No radio continuum emission at $4.8 \mathrm{GHz}$ was detected from IRAS $16272-4837$ up to a $3 \sigma$ limit of $0.2 \mathrm{mJy}$. The 1.2 $\mathrm{mm}$ continuum observations show strong dust emission arising from a region with an elongated morphology, with major and minor axes of $41 . " 0$ and 24 ".7. The observed flux density at $1.2 \mathrm{~mm}$ of $13.8 \pm 0.9 \mathrm{Jy}$ implies a mass of $2.0 \times 10^{3} M_{\odot}$. The line observations indicate a molecular gas region with a size of $\sim 0.4 \mathrm{pc}$, a molecular hydrogen density of $\sim 2 \times 10^{5} \mathrm{~cm}^{-3}$, and a rotational temperature of $\sim 27$ $\mathrm{K}$. We suggest that this object corresponds to a dense massive core in a very early evolutionary stage, distinguished by being luminous but not associated with a UC $\mathrm{H}$ II region (e.g., Cesaroni et al. 1994; Hunter et al. 1998; Molinari et al. 1998). The core is being heated by a recently formed massive star embedded at its center, which is still accreting at high rates, the main heating agent being the accretion luminosity, and has not yet produced a detectable UC H II region. As such, this will become a key object for the study at submillimeter wavelengths with high angular resolution of the earliest stages of the formation of massive stars and their related accretion and outflow processes.

G. G. and D. M. gratefully acknowledge support from the Chilean Centro de Astrofísica FONDAP 15010003. M. G. B. acknowledges support from the Australian Research Council.

\section{REFERENCES}

Bourke, T. L., Garay, G., Lehtinen, K. K., Köhnenkamp, I., Launhardt, R., Nyman, L.-A., May, J., Robinson, G., \& Hyland, A. R. 1997, ApJ, 476, 781

Bronfman, L., Nyman, L.-Å., \& May, J. 1996, A\&AS, 115, 81

Casoli, F., Dupraz, C., Gerin, M., Combes, F., \& Boulanger, F. 1986, A\&A, 169, 281

Cesaroni, R., Churchwell, E., Hofner, P., Walmsley, C. M., \& Kurtz, S. 1994, A\&A, 288, 903

Chini, R., Krügel, E., \& Wargau, W. 1987, A\&A, 181, 378

Churchwell, E. 1990, A\&A Rev., 2, 79

De Pree, C. G., Rodríguez, L. F., \& Goss, W. M. 1995, Rev. Mexicana Astron. Astrofis., 31, 39

Draine, B. T., \& Lee, H. M. 1984, ApJ, 285, 89

Egan, M. P., Shipman, R. F., Price, S. D., Carey, S. J., Clark, F. O., \& Cohen, M. 1998, ApJ, 494, L199

Garay, G., \& Lizano, S. 1999, PASP, 111, 1049

Garay, G., Rodríguez, L. F., Moran, J. M., \& Churchwell, E. 1993, ApJ, 418,368

Gordon, M. A. 1995, A\&A, 301, 853

Hunter, T. R., Neugebauer, G., Benford, D. J., Matthews, K., Lis, D. C., Serabyn, E., \& Phillips, T. G. 1998, ApJ, 493, L97

MacLaren, I., Richardson, K. M., \& Wolfendale, A. W. 1988, ApJ, 333, 821

Mardones, D. 1998, Ph.D. thesis, Harvard Univ., Cambridge

Mardones, D. Myers, P. C., Tafalla, M., Wilner, D. J., Bachiller, R., \& Garay, G. 1997, ApJ, 489, 719

Molinari, S., Brand, J., Cesaroni, R., \& Palla, F. 1996, A\&A, 308, 573

Molinari, S., Brand, J., Cesaroni, R., \& Palla, F. 2000, A\&A, 355, 617

Molinari, S., Brand, J., Cesaroni, R., Palla, F., \& Palumbo, G. G. C. 1998 A\&A, 336, 339

Myers, P. C., Mardones, D., Tafalla, M., Williams, J. P., \& Wilner, D. J. 1996, ApJ, 465, L133

Norberg, P., \& Maeder, A. 2000, A\&A, 359, 1025

Osorio, M., Lizano, S., \& D’Alessio, P. 1999, ApJ, 525, 808

Ossenkopf, V., \& Henning, Th. 1994, A\&A, 291, 943

Price, S. D. 1995, Space Sci. Rev., 74, 81

Sault, R. J., Teuben, P. J., \& Wright, M. C. H. 1995, in ASP Conf. Ser. 77, Astronomical Data Analysis Software and Systems IV, ed. R. A. Shaw, H. E. Payne, \& J. J. E. Hayes (San Francisco: ASP), 433

Sridharan, T. K., Beuther, H., Schilke, P., Menten, K. M., \& Wyrowski, F. 2002, ApJ, 566, 931

Stahler, S. W., Palla, F., \& Ho, P. T. P. 2000, in Protostars and Planets IV, ed. V. Mannings, A. P. Boss, \& S. S. Russell (Tucson: Univ. Arizona Press), 327

van der Tak, F. F. S., van Dishoeck, E. F., Evans, N. J., II, \& Blake, G. A. 2000, ApJ, 537, 283

Walmsley, M. 1995, Rev. Mexicana Astron. Astrofis. Ser. Conf., 1, 137

Walsh, A. J., Burton, M. G., Hyland, A. R., \& Robinson, G. 1998, MNRAS, 301, 640

Walsh, A. J., Hyland, A. R., Robinson, G., \& Burton, M. G. 1997, MNRAS, 291, 261

Wood, D. O. S., \& Churchwell, E. 1989, ApJ, 340, 265

Yorke, H. W. 1984, in Proc. Workshop on Star Formation, ed. R. D. Wolstencroft (Edinburgh: Royal Obs.), 63 